\title{
Transient Electrical Coupling Delays the Onset of Chemical Neurotransmission at Developing Synapses
}

\author{
Theresa M. Szabo, ${ }^{1}$ Donald S. Faber, ${ }^{1}$ and Mark J. Zoran ${ }^{2}$ \\ ${ }^{1}$ Department of Neuroscience, Albert Einstein College of Medicine, Yeshiva University, Bronx, New York 10465, and ${ }^{2}$ Department of Biology, Texas A \& M \\ University, College Station, Texas 77843
}

\begin{abstract}
The formation and subsequent elimination of electrical coupling between neurons has been demonstrated in many developing vertebrate and invertebrate nervous systems. The relationship between the disappearance of electrical synaptic connectivity and the appearance of chemical neurotransmission is not well understood. We report here that identified motoneurons from the snail Helisoma formed transient electrical and chemical connections during regeneration both in vivo and in vitro. Electrical connections that formed in vivo were strongest by day 2 and no longer detectable by day 7. During elimination of this electrical connection, an inhibitory chemical connection from 110 onto 19 formed. This sequence of synaptic development was recapitulated in cell culture with a similar time course. The relationship between the appearance of transient electrical coupling and its possible effects on the subsequent chemical synaptogenesis were examined by reducing transient intercellular coupling. Trophic factor-deprived medium resulted in a $66 \%$ reduction in coupling coefficient. In these conditions, the unidirectional chemical connection formed readily; in contrast, chemical synaptogenesis was delayed in cell pairs exposed to trophic factors where transient electrical coupling was strong. Dye coupling and synaptic vesicle cycling studies supported electrophysiological results. Exposure to cholinergic antagonists, curare and hexamethonium bromide, which block chemical neurotransmission in these synapses, resulted in prolonged maintenance of the electrical connection. These studies demonstrated an inverse relationship between chemical and electrical connectivity at early stages of synaptic development and suggest a dynamic interaction between these forms of neuronal communication as adult neural networks are constructed or regenerated.
\end{abstract}

Key words: electrical synapse; chemical synapse; Helisoma; synaptogenesis; trophic; neurotransmitter

\section{Introduction}

Transient interneuronal communication forms during development before the establishment of adult patterns of synaptic connectivity in many animal systems. Short-lived electrical (and biochemical) coupling is common during both neural development and regeneration (Connors et al., 1983; Peinado et al., 1993a,b; Chang et al., 1999), as is the expression of specific gap junction proteins (connexins; Dermietzel et al., 1989; Guthrie and Gilula, 1989; Lo Turco and Kriegstein, 1991; Nadarajah et al., 1996; Bittman et al., 1997; Owens and Kriegstein, 1998; Rozental et al., 1998; Nadarajah and Parnavelas, 1999). This gap junctional connectivity decreases at developmental times of increased neuronal activity (Kandler and Katz, 1998) and, in some cases, is crucial for chemical synaptogenesis; e.g., in Drosophila visual system (Curtin et al., 2002) and mouse motor neurons (Personius et al., 2001; Personius and Balice-Gordon, 2001). In addition, blockade of chemical synaptogenesis increases both the incidence and dura-

Received Sept. 24, 2003; revised Nov. 2, 2003; accepted Nov. 5, 2003.

This work was supported by National Science Foundation Grant IBN 9421372 (M.J.Z.). We thank Alberto Pereda for critical reading of this manuscript and helpful discussion and comments. Image analysis was conducted in the Cellular Physiology and Molecular Imaging Laboratory at Texas A \& M University, supported by National Institute of Neurological Disorders and Stroke Grant P01 NS-39546.

Correspondence should be addressed to Dr. Mark J.Zoran, Department of Biology, Texas A \& M University, College Station, TX 77843. E-mail: zoran@mail.bio.tamu.edu.

DOI:10.1523/JNEUROSCI.4336-03.2004

Copyright $\odot 2004$ Society for Neuroscience $\quad$ 0270-6474/04/240112-09\$15.00/0 tion of transient coupling during development (Mentis et al., 2002; Pastor et al., 2003), indicating a mutual inhibitory action between the two types of connections. Understanding the contributions made by each type of communication, as well as why and how nervous systems transition from one type to the other is crucial to understanding the formation of neural networks.

Invertebrate systems have been valuable models for studying mechanisms underlying synapse formation and function, because of the widespread occurrence of both chemical and electrical synaptic connections, along with the fact that many of their neurons are large, visually identifiable, and can be readily isolated into cell culture. Neurons from Aplysia (Bodmer et al., 1988; Carrow and Levitan, 1989), Lymnaea (Janse et al., 1986; Hamakawa et al., 1999a,b; Woodin et al., 1999), the leech (Ready and Nicholls, 1979; Nicholls et al., 1990; Fernandez-de-Miguel and Drapeau, 1995; De-Miguel, 2000; Kristan et al., 2001), and our system, Helisoma trivolvis (Hadley et al., 1982, 1983, 1985; Hadley and Kater, 1983; Kater, 1985; Zoran et al., 1996; Magoski and Bulloch, 1998) have been used extensively for the study of both electrical and chemical synapse formation between identified neurons in vivo and in cell culture.

To directly examine factors affecting the sequential expression of electrical and chemical synapses during development, we used regenerating motoneurons from the snail Helisoma. The capability of these neurons to reproduce the developmental connectivity seen in reduced ganglia preparations at a specific synapse in cell 
culture make them an especially useful model for examination of potential contributing factors. In both regenerating ganglia and cell culture we observed that electrical connections formed first and were then eliminated just before the onset of unidirectional chemical synaptogenesis. Using trophic factor deprivation and antagonists of synaptic transmission, we tested the hypotheses (1) that transient expression of electrical connectivity during early stages of cell-cell contact affects subsequent chemical synaptogenesis, and (2) that the suppression of chemical synaptogenesis influences the formation of electrical coupling. We demonstrate here that junctional coupling and chemical synaptogenesis negatively interact, such that transient electrical synaptogenesis delays the formation of chemical synapses between specific neurons of Helisoma.

\section{Materials and Methods}

Animals and preparations. Experiments were conducted on laboratory stocks of albino (red) pond snails, Helisoma trivolvis, which were maintained in 20 gallon aquaria at $26^{\circ} \mathrm{C}$. Aquaria were kept on a controlled photoperiod of $12 \mathrm{hr}$ light/dark, and animals were fed lettuce and/or trout chow daily.

Adult buccal ganglia. Dissection methods have been described previously (Haydon and Zoran, 1991). Two buccal neurons, both of which innervate radular tensor muscle groups (Kater, 1974; Zoran et al., 1989), were used for these studies: neurons 19 and 110 (previously B19 and VB10, respectively; see Murphy, 2001). They were identified based on their size, position, and pigmentation. Experiments using isolated buccal ganglia were performed in defined medium (DM) (Poyer and Zoran, 1996). Before electrophysiological recording or dye injection, preparations were pinned onto a Sylgard-coated glass Petri dish containing $2 \mathrm{ml}$ of saline with $10 \times$ normal calcium (in mM: $40.0 \mathrm{NaCl}, 1.7 \mathrm{KCl}, 41.0$ $\mathrm{CaCl}_{2}, 1.5 \mathrm{MgCl}_{2}$, and 10.0 HEPES, pH 7.5) to reduce motor activity.

Regenerating buccal ganglia. Intact Helisoma were placed in a saturated menthol solution for 15-30 min. Suction was gently applied to the ventral portion of the foot to extract the anterior region of the body from the shell. A dorsal incision was made, the buccal commissure was crushed with fine forceps, and the body wall was sutured. Up to $7 \mathrm{~d}$ were allowed for recovery, after which ganglia were removed, and synaptic connectivity was assessed. Sham operations involving surgery preparations without commissural crush demonstrated no transient connectivity between buccal neurons (data not shown).

Cell culture. Identified buccal neurons were isolated into culture as previously described (Haydon and Zoran, 1991; Poyer and Zoran, 1996). Neurons were transferred to $35 \mathrm{~mm}$ culture dishes (No. 1008 Falcon) made non-adhesive by pretreatment with a $0.5 \%$ solution of bovine serum albumin (BSA) and containing $2 \mathrm{ml}$ of conditioned medium (CM). $\mathrm{CM}$ was generated by incubating two central ring ganglia per $1 \mathrm{ml}$ of DM in a glass culture dish (silicone-treated) for $3 \mathrm{~d}$. Neurons were cultured for $3 \mathrm{~d}$ in $\mathrm{CM}$ on a nonadhesive substrate to allow for retraction of neuronal processes and the formation of spherical somata. They were then transferred into experiment-specific culture conditions and manipulated into cell-cell contact. After $0-5 \mathrm{~d}$ of contact, cell pairs were transferred to recording chambers [poly-L-lysine (PLL)-coated dishes containing $2 \mathrm{ml}$ of DM] for electrophysiological study. All cultures were incubated at $25^{\circ} \mathrm{C}$.

Electrophysiological techniques. Electrophysiological analyses were performed using intracellular recording techniques. Glass microelectrodes (borosilicate; FHC) were filled with $1.5 \mathrm{M} \mathrm{KCl}$ and possessed tip resistances ranging from $10-20 \mathrm{M} \Omega$. Current-clamp recordings of neuronal membrane potentials were amplified using bridge-balanced electrometers (Getting Instrumental Inc.). Simultaneous recordings were made from 19-19, 110-110, and 19-110 cell pairs. Typical resting potential for these cells are $-60 \mathrm{mV}(110)$ and $-70 \mathrm{mV}$ (19). In instances in which either cell was spontaneously active, its membrane potential was maintained subthreshold (near $-70 \mathrm{mV}$ ) with base current injections. Electrophysiological signals were digitized by a MacLab analog-to-digital data acquisition system linked to a Macintosh computer using Chart software.

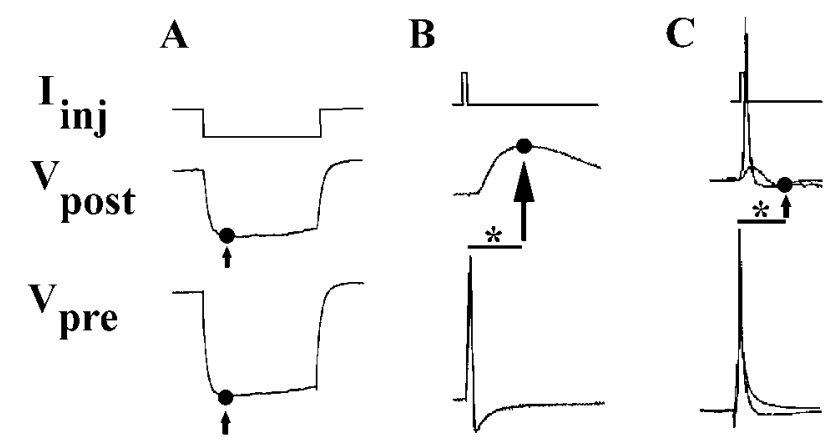

Figure 1. Quantification and assessment of synaptic connectivity. Connectivity between two neurons was determined by injecting current $\left(l_{\text {inj }}\right)$ into the presynaptic cell, while recording changes in the membrane potential of both that cell $\left(V_{\text {pre }}\right)$ and the postsynaptic $\left(V_{\text {post }}\right)$ one. $A$, Hyperpolarizing current injection into one cell of an electrically coupled pair resulted in hyperpolarizing presynaptic and postsynaptic voltage changes. Electrical coupling coefficients were determined using voltages measured at points of peak membrane hyperpolarization $(\boldsymbol{O}$, indicated by arrow). $B, C$, Depolarizing current injection into a presynaptic neuron resulted in presynaptic APs (bottom traces) and PSPs (middle traces). PSPs at these mixed synapses could contain electrical and/or chemically based components. To distinguish these components, PSP amplitude in the postsynaptic cell was assessed at $95 \mathrm{msec}\left({ }^{*}\right.$, scale bar) after the peak of the presynaptic AP. Time points used for voltage measurement ( $\boldsymbol{O}$, indicated by arrows) identified postsynaptic responses that range from mostly chemical $(B)$ to mostly electrical $(C)$.

Electrical coupling was measured by injection of constant amplitude, hyperpolarizing current pulses $(0.5-1 \mathrm{nA}$ pulse for $3 \mathrm{sec}$ ) into one neuron of a pair while recording membrane voltage changes in both the presynaptic (injected) neuron and the postsynaptic (non-injected) partner (Fig. $1 A$ ). The presynaptic signal was usually $30-50 \mathrm{mV}$ in amplitude. Electrical coupling coefficients (ECCs) were determined as the ratio of postsynaptic to presynaptic voltage changes: ECC $=\Delta V_{\text {post }} / \Delta V_{\text {pre }}$ (Bennett, 1977). Data analyses for coupling ratios and input resistance measurements were taken at the point of maximal membrane hyperpolarization (Fig. $1 \mathrm{~A}$, arrows). Analysis of unidirectional coupling data determined that electrical connections formed between all neuronal pairs were nonrectifying. Thus, all analyses presented were averages of bidirectional data and a single mean coupling coefficient was calculated for each pair.

An analysis of chemical connections from neuron 110 onto neuron 19 was conducted by injection of suprathreshold depolarizing current into presynaptic neurons. While simultaneously recording voltage changes in presynaptic and postsynaptic neurons (Fig. $1 \mathrm{~B}, \mathrm{C}$ ), four to five trains of 10 action potentials (APs) (one per second) were evoked, and postsynaptic potentials (PSPs) in neuron 19 were examined. Spontaneous synaptic potentials (SSPs), most common in the first $12 \mathrm{hr}$ in culture, were monitored for $2 \mathrm{~min}$. Their presence was not a predictor of evoked chemical synaptic transmission. Because many soma-soma synapses possessed both electrical and chemical components, criteria were developed to distinguish between electrical and chemical contributions. An analysis of $110-19$ preparations possessing exclusively chemical connectivity (using acetylcholine receptor antagonists and age-specific synapses; see Results) indicated that the average latency from AP peak to PSP peak was $95 \mathrm{msec}$ (Fig. $1 B$ ). In preparations possessing exclusively electrical coupling, postsynaptic membrane voltage returned to resting values by $95 \mathrm{msec}$ (Fig. 1C). In this analysis, even cell pairs possessing large ECC values (0.4-0.8) exhibited essentially no PSP at $95 \mathrm{msec}(0.1 \pm 0.1 \mathrm{mV} ; n=8)$. Therefore, a time point of $95 \mathrm{msec}$ after the peak of the presynaptic action potential was used in all chemical transmission analyses. Stimulation of neuron 19, in contrast to presynaptic 110, never evoked chemical PSPs in postsynaptic neurons, as determined by cholinergic blocker studies and membrane potential manipulations.

To examine the possible impact of electrical coupling on postsynaptic membrane potential at $95 \mathrm{msec}$, and therefore the degree to which these connections could impact the assessment of chemical synaptic connectivity (PSP amplitude) at this time, we estimated the peak coupling 
potential that the presynaptic spike, $V_{\mathrm{AP}}$, would produce in a postsynaptic cell as:

$$
V_{\mathrm{AP}}{ }^{\star} \operatorname{ECC}_{\mathrm{SS}}\left(1-e^{-\mathrm{ts} / \tau}\right),
$$

where,

$\mathrm{ECC}_{\mathrm{s}}=$ electrical coupling coefficient at steady state, $t_{\mathrm{s}}=$ duration of the AP as determined by the AP half-width, and $\tau=$ time constant of the postsynaptic potential change in response to presynaptic current injection.

Then, the predicted electrotonic coupling potential, or PSP, $95 \mathrm{msec}$ after the AP peak would be:

$$
V_{\mathrm{P}}=\left(V_{\mathrm{AP}}{ }^{\star} \mathrm{ECC}_{\mathrm{SS}}\right)\left(1-e^{-\mathrm{ts} / \tau}\right)\left(e^{-95 / \tau}\right) .
$$

Membrane voltage changes during hyperpolarizing current injection were used to empirically determine $\tau$ for postsynaptic neurons, and $V_{\mathrm{p}}$ values were then compared to experimentally obtained PSPs (see Results).

It was possible that chemical PSPs would not be detected because of a low driving force; that is, a reversal potential close to resting potential. To guard against this possibility, postsynaptic membrane potential was varied, in both the hyperpolarizing and depolarizing directions, wherever there was no obvious PSP.

Cellular imaging techniques. For fluorescent imaging studies, neurons were isolated and paired as described for electrophysiological studies. Cells were transferred to DM-containing adhesive (PLL-coated) glassbottomed dishes and visualized using phase-contrast and epifluorescence optics. Images were captured and analyzed using a Zeiss (Oberkochen, Germany) Axioplan microscope, Hamamatsu (Bridgewater, NJ) C5810 camera, and Adobe Photoshop software (Adobe Systems, San Jose, CA). A portion of the image devoid of cells, processes, or debris was sampled for dark background (DB) subtraction.

The tracer Neurobiotin (NB) (Vector Laboratories, Burlingame, CA) was used to assess dye coupling between neurons. NB was pressureinjected into a neuron $(10-15 \mathrm{msec}$ pulses at $30 \mathrm{psi})$ and then allowed 1 $\mathrm{hr}$ to diffuse in coupled partners before being fixed for $5 \mathrm{~min}$ in $4 \%$ paraformaldehyde in PBS. Mean fluorescence intensity values were obtained from images, and dye coupling coefficients (DCCs) were determined as the ratio fluorescence intensity of the non-injected (postsynaptic) neuron to that of the injected (presynaptic) neuron.

The styryl dye FM1-43 (Molecular Probes, Eugene, OR) was used to monitor synaptic vesicle recycling (Betz et al., 1992; Diefenbach et al., 1999; Kay et al., 1999). During the "staining" portion of the protocol, 25 $\mu \mathrm{M}$ FM1-43 in DM was bath-applied in high- $\mathrm{K}^{+}$saline $(50 \mathrm{~mm} \mathrm{KCl})$ for $1 \mathrm{~min}$. Excess dye was removed via flow-through exchange with a $20 \mathrm{ml}$ wash of zero $\mathrm{Ca}^{2+} \mathrm{DM}\left(4.1 \mathrm{mM} \mathrm{Ca}^{2+}\right.$ replaced by $\left.10 \mathrm{mM} \mathrm{Mg}^{2+}\right)$. During the "destaining" portion of the protocol, high- $\mathrm{K}^{+}$saline without FM1-43 was bath-applied. Bath application of high- $\mathrm{K}^{+}$saline would presumably stimulate both neurons whether coupled or not, and in this way control for differences in partner activity. Fluorescence intensities were determined by measuring the brightness of the area encompassing the entire cell pair.

Receptor antagonist studies. Neurons 19 and 110 are both motoneurons with some common targets. Neuron 19, like many other Helisoma motor neurons, is cholinergic at its neuromuscular synapse (Poyer and Zoran, 1996). To determine the nature of the chemical connection from neuron 110 onto neuron 19 , studies were performed using the competitive cholinergic antagonists D-tubocurarine chloride (curare; D-TC) and hexamethonium bromide (Hex) in DM. Neurons 19 and 110 were cultured for $24 \mathrm{hr}$ in DM to enhance the presence of the chemical connection. A 10 $\mu \mathrm{M}$ solution of D-TC and Hex were bath-applied to acutely block neurotransmission at these synapses. For chronic studies, cells were cultured for $24 \mathrm{hr}$ in $2 \mathrm{ml} \mathrm{CM}$ containing $10 \mu \mathrm{M} \mathrm{D}-\mathrm{TC}, 10 \mu \mathrm{M} \mathrm{Hex}$, or $10 \mu \mathrm{M}$ vehicle.

Data analysis. Two-tailed Student's $t$ tests or ANOVA were used for statistical analysis. Data are presented as mean plus or minus SEM, unless otherwise indicated.

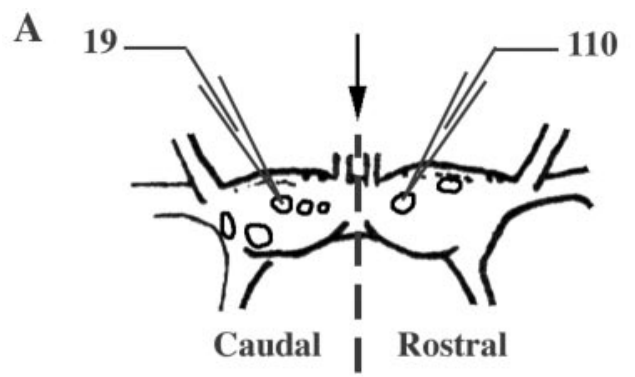

B
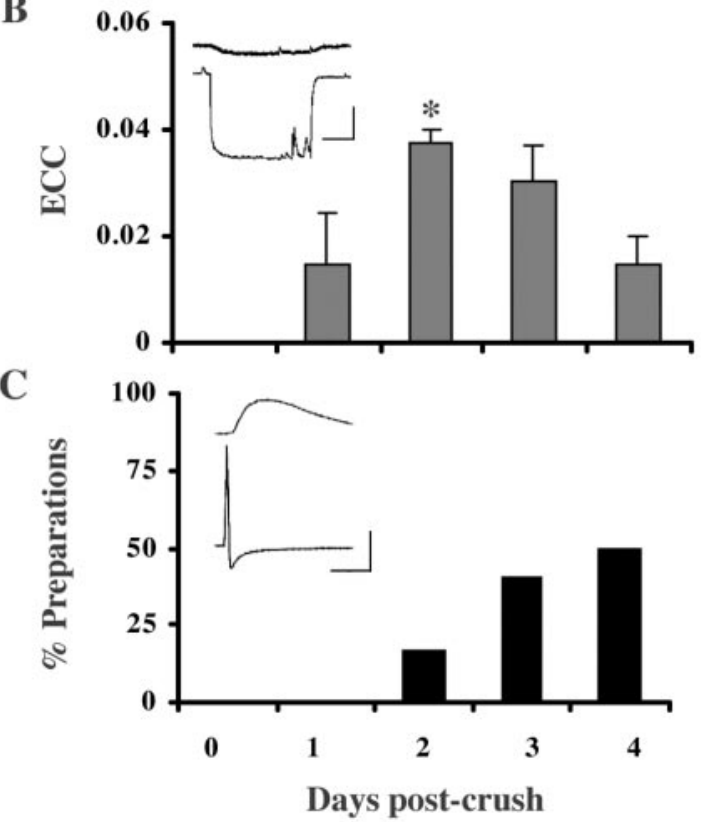

Figure 2. Neurons 19 and 110 developed electrical and chemical connections in regenerating buccal ganglia in vivo. $A$, Diagrammatic buccal ganglia illustrating the site of commissural crush (arrow) as well as the caudal 19 and the rostral 110. B, C, Temporal sequence of synapse formation between neurons 110 and 19 during regeneration in vivo. Simultaneous electrophysiological recordings in neurons 19 and 110 were performed from 0 to $4 \mathrm{~d}$ of regeneration (post-crush). B, These cells possessed no connectivity (electrical or chemical) in adult ganglia before commissural crush (day 0). By day 2 of regeneration, 19 and 110 possessed peak electrical coupling, which was significantly reduced by day 4 (ANOVA; $p=0.13$; post hoc LSD; ${ }^{*} p<$ $0.05)$. Data represent mean $\pm S E M$. Inset, Representative recording depicting electrical coupling between neurons 110 (bottom trace) and 19 (top trace) on day 2. Horizontal bar equals 1 $\mathrm{sec}$, vertical bar equals $20 \mathrm{mV}$ for the presynaptic trace, $10 \mathrm{mV}$ for the postsynaptic trace. C, Chemical synaptic connectivity developed more slowly, with the greatest number of preparations demonstrating chemical connections on day 4. (For chemical and electrical analysis: $d 1$, $n=7 ; \mathrm{d} 2, n=6 ; \mathrm{d} 3, n=5 ; \mathrm{d} 4, n=6$ ). Inset, Representative recording demonstrating a chemical PSP in neuron 19 (top trace) in response to an evoked AP in neuron 110 (bottom trace). Horizontal bar equals $1 \mathrm{sec}$, vertical bar equals $20 \mathrm{mV}$ for the presynaptic trace (neuron 110), 10 $\mathrm{mV}$ for the postsynaptic trace (neuron 19).

\section{Results}

Helisoma buccal ganglia possess neurons that are visually identifiable by their size, pigmentation, and position relative to other neurons (Kater, 1974). Motor neurons in these ganglia control movements of the feeding musculature and associated structures, namely, the radula, odontophore, and corresponding tensor muscles. Neuron 19, a hyperretractor motor neuron, and neuron 110 , a retractor motor neuron (Murphy, 2001), are readily accessible electrophysiologically with intracellular recording electrodes through the ganglion sheath (Fig. 2A). Bilateral left and right neurons 19 were electrically coupled in adult ganglia with an average coupling coefficient of $0.22 \pm 0.02(n=12)$, a value similar to that reported previously for these synapses (Goldberg 
and Kater, 1989). Neurons 19 and 110 did not possess electrical or chemical synaptic connectivity with each other in adult ganglia, as indicated by electrophysiology (Fig. $2 B, C$, Day 0$)(n=6)$ and neurobiotin dye-coupling experiments (data not shown).

\section{Synaptic connections between 19 and 110 form during regeneration}

Previous studies in Helisoma demonstrated that commissural crush (Fig. $2 \mathrm{~A}$, arrow) can result in the formation of novel interneuronal connections during regeneration (Hadley et al., 1982). The commissure connecting the right and left buccal ganglia contains the axonal processes of many neurons, including those of 19 and 110 . Because neurons 19 and 110 are not synaptically connected in adult Helisoma, their connectivity during regeneration was examined to assess their ability to form novel connections. Neuron 19 possesses a constrained capacity for chemical synapse formation (Poyer and Zoran, 1996) but readily forms novel electrical synapses during regeneration in vivo and in cell culture (Hadley et al., 1983). Synapse formation by neuron 110 has not been described.

After commissural crush, a bidirectional electrical connection formed between neurons 19 and 110 in 100\% of preparations by day 2 of regeneration $(\mathrm{ECC}=0.04 \pm 0.01 ; n=6)$ (Fig. $2 B$, inset), and it was only weakly detectable by day $4(n=6)$. As electrical coupling declined, a unidirectional chemical connection from neuron 110 onto neuron 19 was detected in $16.7 \%$ of preparations on day $2(n=6)$ and $50 \%$ of preparations by day $4(n=6)$ (Fig. 2C, inset). Chemical connectivity from 19 onto 110 was never detected ( $n=6$; data not shown). Thus, within $4 \mathrm{~d}$ of axonal injury, an electrical connection between neurons 110 and 19 formed and was then eliminated, while a chemical synapse from 110 onto 19 gradually emerged that was detected in $66.7 \%$ of preparations by day 7 ( $n=6$; data not shown). By 5 weeks, there is evidence that these connections are lost $(n=2)$.

\section{Transient 19-110 synaptic connectivity is formed in cell culture}

To determine whether these results could be recapitulated in cell culture and therefore examined in a more controlled environment, neurons 19 and 110 were isolated, manipulated into contact, and paired for an additional 1 or $5 \mathrm{~d}$ (Fig. $3 A$ ). The giant somatic synapses formed in these experiments (Fig. 3B) were advantageous because the geometry of the soma-soma preparation allowed for efficient detection of synaptic potentials with recordings performed at sites immediately adjacent to synaptic contacts. In addition, the onset and duration of cell-cell contact could be strictly controlled at these somatic synapses. By day 5 in culture, the process of somatic outgrowth in the absence of an adhesive substrate generated a mass, or fascicle, between neurons that forced the two cell bodies apart (Fig. $3 B$ ). Cells paired in DM, media lacking trophic factors that promote outgrowth, did not produce fascicular masses (data not shown).

Somatic 19-110 pairs formed electrical connections possessing an average ECC that equaled $0.51 \pm 0.06(n=14)$ after $1 \mathrm{~d}$ of contact and decreased significantly by day 5 to $0.15 \pm 0.02(n=$ $18 ; p<0.001)$. In contrast, $19-19$ and $110-110$ somatic pairs formed electrical connections by day 1 that were maintained or slightly strengthened over this $5 \mathrm{~d}$ period (Fig. 3C). Electrical coupling was virtually identical across synaptic groups on day 1 . As is the case in adult ganglia, chemical connectivity was never detected in 19-19 or 110-110 pairs in cell culture. However, a unidirectional chemical connection from neuron 110 onto neuron 19 was detected by day 5 of contact in $83 \%$ of preparations,

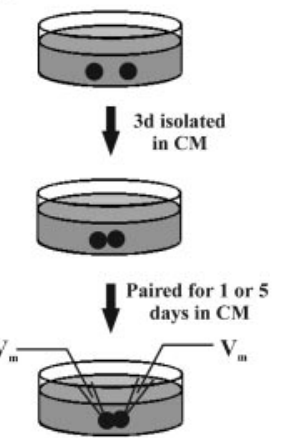

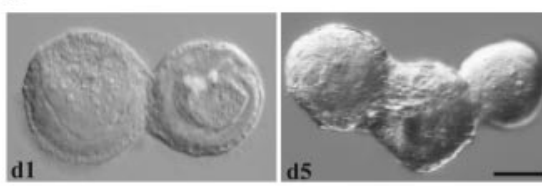

C

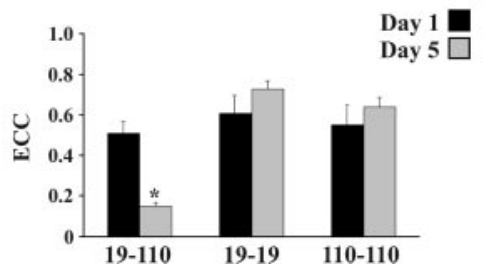

Figure 3. Neurons 19 and 110 cultured as spherical somata in non-adhesive conditions formed strong synaptic contacts and exhibited fascicular growth. A, All neurons were cultured alone in $\mathrm{CM}$ for $3 \mathrm{~d}$ before experimental manipulation to allow for process retraction and the formation of a uniform spherical morphology. Neurons 19 and 110 were then paired into contact in fresh CM, in nonadhesive culture dishes, for 1 or $5 \mathrm{~d}$. B, After $24 \mathrm{hr}$ of contact (d1), soma-soma pairs formed strong, adhesive connections that could not be disrupted without cellular damage. By day 5 of contact (d5), neurons displayed growth of a fascicular mass between somatic partners. Scale bar, $10 \mu \mathrm{m}$. C, Neurons 19 and 110 formed synaptic connections in a partner-specific manner. After $1 \mathrm{~d}$ paired in cell culture, $19-19(n=12), 110-110(n=$ $11)$, and $19-110$ ( $n=14$ ) all formed strong electrical connections. After $5 \mathrm{~d}$ paired in culture, $19-19(n=13)$ and $110-110(n=9)$ ECC values are not significantly different than on day 1. In contrast, $5 \mathrm{~d} 19-110 \mathrm{ECC}$ values were significantly lower than day 1 values $\left({ }^{*} n=18 ; p<\right.$ 0.0001).

but never from neuron 19 onto neuron $110(n=6)$. Electrophysiologically, these chemical connections are virtually indistinguishable from those formed during regeneration in vivo (Fig. 2C). These results demonstrated that neurons 19 and 110 formed, maintained, and eliminated electrical and unidirectional synaptic connections in cell culture over a time course similar to that observed in ganglia regenerating in vivo.

\section{Ganglia-derived trophic factors affect electrical synapse formation}

To further examine the relationship between transient electrical and chemical synapse formation, we used established pharmacological blockers of electrical coupling. These included octanol, which often resulted in loss of cell-cell adhesion in these preparations, snail hemolymph, and $18-\alpha$-glycyrrhetinic acid (AGA) (Bennett, 1997). Although AGA did successfully reduce the strength of electrical synapses between 19-19 and 19-110 neuronal pairs, it also significantly reduced spontaneous release rates in these neurons, and its actions were highly variable between trials. In addition, control experiments using DMSO vehicle alone (in which AGA was solubilized) resulted in significant reductions in electrical coupling at these synapses. These agents were therefore determined to be unsatisfactory tools in our cell culture preparations.

Previous studies in Helisoma demonstrated that the formation of electrical synapses is linked to the process of neurite extension (Hadley and Kater, 1983; Hadley et al., 1985) and that, in the absence of conditioning factors (i.e., in DM) cultured neurons did not extend neurites. We therefore paired cells in DM to determine whether deprivation of trophic factors would result in reduced electrical coupling. To determine whether overall cellular physiology was altered under these conditions, spontaneous synaptic potential (SSP) rates were examined as well as other biophysical properties including membrane resistance, spike height, and spike half-width. None of these characteristics dif- 
A
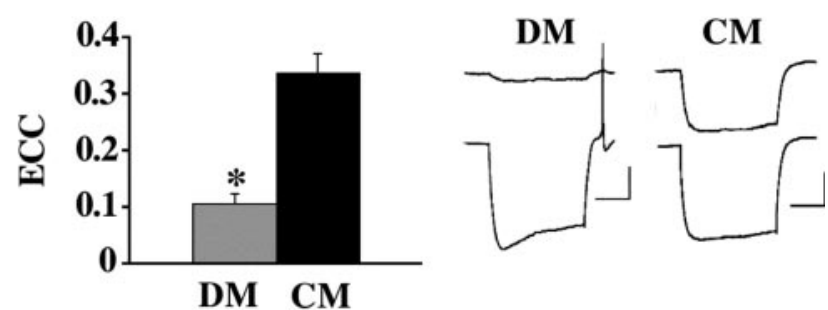

B

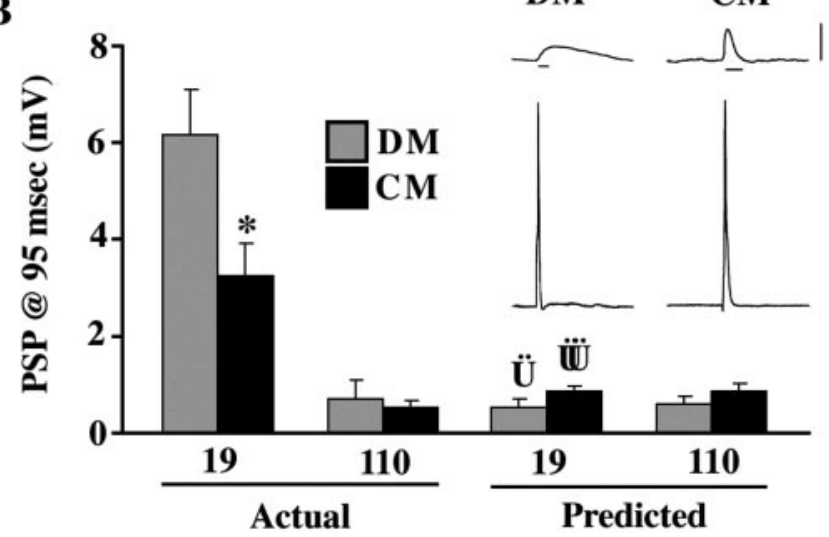

Figure 4. Chemical and electrical synaptic connectivity at 19-110 neuronal contacts were inversely correlated. $A$, Neurons 19 and 110 were paired for $24 \mathrm{hr}$ in the presence $(C M)$ or absence (DM) of trophic factors. Neuronal pairs in CM $(n=38)$ possessed ECCs significantly greater than those of cells paired in DM $\left(n=20 ;{ }^{*} p<0.0001\right)$. Data represent average bidirectional ECC values for each cell pair. Traces illustrate electrical coupling at representative 19-110 pairs where hyperpolarizing current was injected into neuron 110 (bottom trace). Horizontal bar equals $1 \mathrm{sec}$, vertical bar equals $20 \mathrm{mV}$. B, Actual, PSP amplitudes at $95 \mathrm{msec}$ in neuron 19 in response to evoked APs in neuron 110 were significantly greater in DM $(n=20)$ than in $\mathrm{CM}(n=38)$ after $24 \mathrm{hr}$ of contact $\left({ }^{*} p<0.005\right)$. In contrast, chemical synaptic connections from neuron 19 onto neuron 110 were virtually absent in these pairs. Labels (19 and 110) refer to the postsynaptic cell. Predicted PSP amplitudes at $95 \mathrm{msec}$ for postsynaptic neuron 19 and postsynaptic neuron $110\left(V_{p}\right)$ were calculated using biophysical measurements (including ECC and $\tau$ ) taken in DM and CM (see Materials and Methods). $V_{\mathrm{p}}$ values for postsynaptic 110 in CM and DM were not different from actual PSP values taken at $95 \mathrm{msec}(p>0.05 ;(M$ and DM), and was therefore representative of synapses with no chemical synaptic connections. $V_{\mathrm{p}}$ values for postsynaptic 19 in CM and DM were significantly different from actual PSP values taken at 95 $\operatorname{msec}(\ddot{\mathbf{U}} p<0.005 ; \ddot{\mathbf{U}} p<0.005$ ), demonstrating that these voltage changes due to electrical coupling alone did not account for voltage differences at $95 \mathrm{msec}$ post AP. Inset, Traces show a PSP in neuron 19 in response to an evoked AP in neuron 110 in either DM or CM. Horizontal bar equals $95 \mathrm{msec}$, vertical bar equals $20 \mathrm{mV}$ for presynaptic (bottom) traces, $10 \mathrm{mV}$ for postsynaptic (top) traces.

fered significantly for neuron 19 or 110 when DM and CM culture groups were compared.

Electrical coupling between neurons 19 and 110 paired for 24 $\mathrm{hr}$ in DM possessed significantly lower ECC values than cells paired in CM $(p<0.001)$ (Fig. $4 A)$. To determine whether this threefold reduction in electrical coupling affected chemical synaptogenesis, PSP amplitude was assessed after $1 \mathrm{~d}$ of contact in $\mathrm{CM}$ and DM. The amplitude of AP-evoked PSPs was significantly higher in $\mathrm{CM}(p<0.005)$ (Fig. $4 B)$.

To determine whether electrical coupling might complicate the assessment of PSP amplitude values at $95 \mathrm{msec}$, the contribution of an electrically based PSP to membrane depolarization at 95 msec was empirically determined ( $V_{\mathrm{p}}$; see Materials and Methods). $V_{\mathrm{p}}$ values (predicted PSP amplitudes) for postsynaptic 19 were significantly lower than actual PSPs recorded in postsynap-
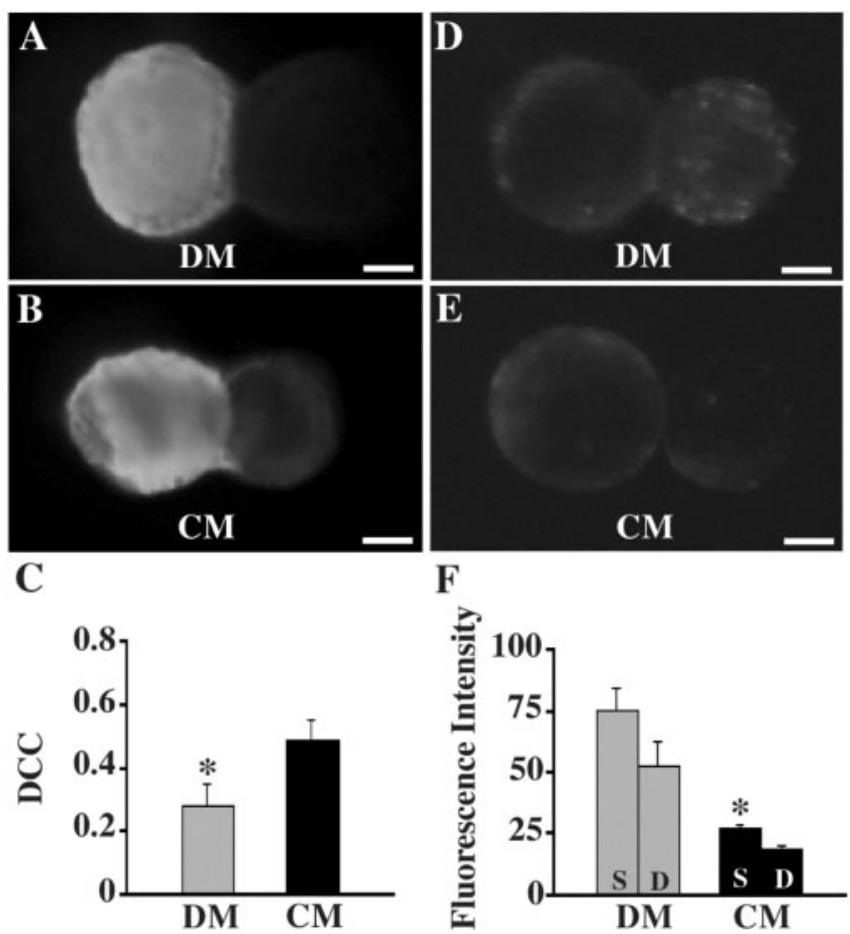

Figure 5. Dye-coupling and vesicle cycling were altered after trophic factor deprivation. 19-110 pairs were cultured for $24 \mathrm{hr}$ in DM or CM, as previously described. For all images, neuron 19 is on the left, and neuron 110 is on the right. $A-C$, Neurobiotin was pressure-injected into neuron 19 and allowed $1 \mathrm{hr}$ to diffuse across junctional contacts. After avidin immunochemistry, fluorescent images were obtained, and DCCs were determined. DM cultured pairs possessed significantly lower DCC values than $C M$ pairs $\left(C ; n=5\right.$ for $D M ; n=10$ for $C M ;{ }^{*} p<$ $0.05) . D-F$, The styryl dye FM1-43 was bath-applied to cell cultures in the presence of high- $K^{+}$ saline, and fluorescent images were obtained ( $D-F ;$ Stain, S). High- $K^{+}$saline alone was again bath-applied (Destain, D), and fluorescence intensity was assessed. DM pairs possessed significantly higher fluorescence intensities than $\mathrm{CM}$ pairs $\left(F ; n=6\right.$ for DM; $n=9$ for $\mathrm{CM}^{*}{ }^{*} p<$ 0.005). Fluorescence staining distributed over the entire somatic surface of neuron 110 in $C M$ indicated that vesicle cycling was not specifically localized to sites of soma-soma contact $(D)$. Scale bars, $10 \mu \mathrm{m}$.

tic 19 at $95 \mathrm{msec}(p<0.005)$ (Fig. $4 B)$. Furthermore, PSP amplitudes in postsynaptic neuron 110, in which chemical synaptic transmission from neuron 19 was never detected, were not significantly different from predicted values $\left(V_{\mathrm{p}}\right)$ based on electrical coupling alone $(p>0.05)$ (Fig. $4 B)$. These results demonstrated that postsynaptic voltage changes caused by electrical coupling did not account for the differences in PSP amplitude in neuron 19 at $95 \mathrm{msec}$ in DM versus $\mathrm{CM}$, and that electrical coupling did not compromise chemical synaptic PSP assessments at this time.

The inverse correlation between electrical and chemical synaptogenesis in 19-110 somatic synapses was also detectable using imaging techniques. Biochemical coupling was examined using the tracer NB. NB coupling between 19-110 neurons after $24 \mathrm{hr}$ in culture demonstrated significantly lower DCC values in DM than in $\mathrm{CM}(p<0.05)$ (Fig. $5 A-C)$. This $40 \%$ reduction in dyecoupling supported electrophysiological results, indicating reduced biochemical coupling in cell pairs deprived of conditioning factors.

Additionally, activity-dependent uptake of the dye FM1-43 at somatic synapses, which is proportional to synaptic vesicle turnover, was greater in DM than CM. 19-110 pairs in DM displayed average fluorescence intensities significantly larger than pairs in $\mathrm{CM}(p<0.003)$ (Fig. $5 D-F)$. Fluorescence intensity decreased in both DM and CM pairs when exposed to destaining stimulation 

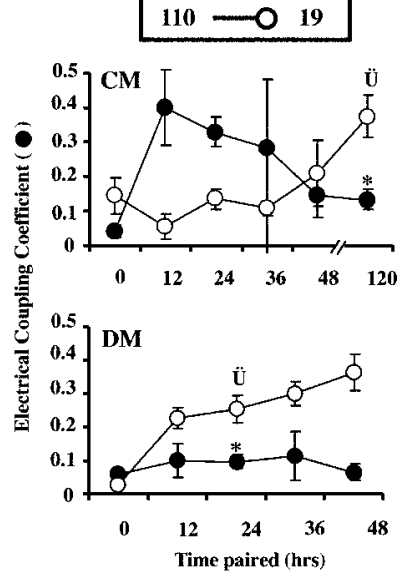

$19-0110$

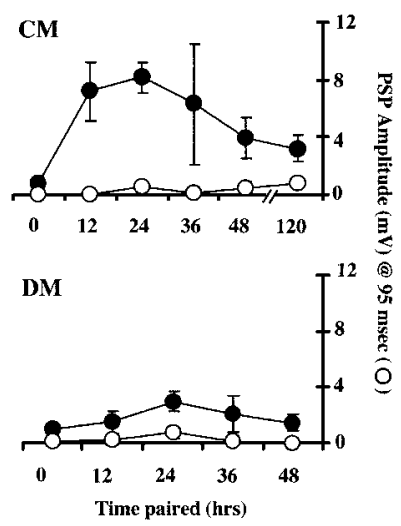

A

B

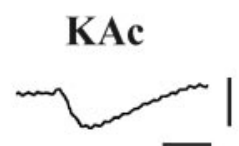

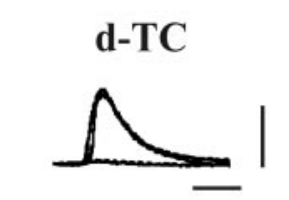

C

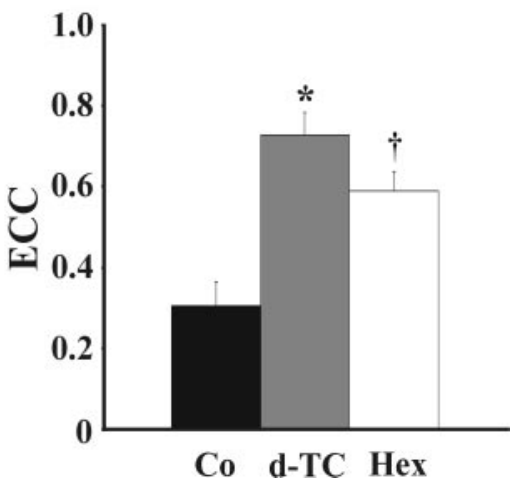

Figure 6. Time course of electrical and chemical synaptogenesis in 19-110 soma-soma pairs in culture. Both $y$-axis labels apply to all four graphs. Current was injected into either presynaptic neuron 110 (left graphs) or presynaptic neuron 19 (right graphs) while membrane potential was simultaneously recorded in both neurons. Left, top, Time course of synapse formation in CM. At 12 and $24 \mathrm{hr}$, in the presence of strong, trophic factor-induced electrical coupling, PSP amplitudes were at their lowest. By $120 \mathrm{hr}$, when ECC values were lower $\left({ }^{*} p<\right.$ 0.0005), PSP amplitudes were significantly elevated above $24 \mathrm{hr}$ levels ( bottom, Time course of synapse formation in DM. In the absence of trophic factors, electrical coupling never developed strongly, and ECC values were significantly lower than those in CM at $24 \mathrm{hr}\left({ }^{*} p<0.0001\right)$. At this same time point, significantly higher PSP amplitudes were seen in DM than in CM ( 0 : $p<05$ ). By $48 \mathrm{hr}$ of contact in DM, ECC values were still low, but PSP amplitudes $(8.7 \pm 1.3 \mathrm{mV}$ ) had increased to values similar to those seen after $5 \mathrm{~d}$ of culture in CM (CM at $120 \mathrm{hr}: 9.0 \pm 1.5 \mathrm{mV})$. Right, top and bottom, Time course of electrical and chemical synapse formation with current injection into neuron 19. ECCs were similar to 110 current injections with strong transient coupling in CM, but not DM. Thus, electrical synaptic connections were non-rectifying in both DM and CM pairs (right vs left graphs of ECC values). PSPs in neuron 110 at 95 msec from AP peak were virtually undetectable in response to stimulation of neuron 19 in both DM and CM. ( $n$ : DM, $0 \mathrm{hr}=16,12 \mathrm{hr}=4,24 \mathrm{hr}=16,36 \mathrm{hr}=9,48 \mathrm{hr}=$ $7 ; C M, 0 \mathrm{hr}=15,12 \mathrm{hr}=6,24 \mathrm{hr}=32,36 \mathrm{hr}=9,48 \mathrm{hr}=6,120 \mathrm{hr}=19$.)

in the absence of FM1-43 dye (Fig. 5F). Somatic synapses cultured in DM incorporated approximately three times as much dye as synapses in CM. This additional fluorescence was widely accounted for by increased punctate staining in neuron 110 (Fig. $5 D$ ), supporting electrophysiological findings that DM pairs possess a greater capacity for chemical synaptic transmission than CM pairs.

\section{Electrical and chemical transmission are inversely related}

To temporally examine the inverse relationship between electrical and chemical synapse formation, 19-110 cell pairs were cultured in DM or CM, and their synaptic connectivity was examined at $0,12,24,36$, and $48 \mathrm{hr}$ after onset of contact. At 12 and 24 hr of contact in CM, 19-110 soma-soma pairs exhibited strong electrical coupling, whereas chemical connectivity was weak (Fig. 6 , top left). DM-cultured pairs at these same time points (12-24 hr) possessed lower ECCs and higher PSP amplitudes than CMcultured cell pairs (Fig. 6, bottom left). Ultimately, PSP amplitudes reached equivalent levels in the two conditions (Fig. 6, $48 \mathrm{hr}$ $\mathrm{DM}$ vs $120 \mathrm{hr}$ CM). Thus, the presence of transient electrical coupling was correlated with a $72 \mathrm{hr}$ delay in the maturation of chemical synaptic transmission. Figure 6 also confirms the bilateral symmetry in ECC (top, left versus right) and the lack of chemical connectivity from neuron 19 onto 110 at any time in cell culture (right, top and bottom).
Figure 7. Blockade of cholinergic communication in 19-110 pairs resulted in enhanced electrical synaptic communication. $A$, Neurotransmitter release from neuron 110 onto neuron 19 is cholinergic. For cell pairs possessing strictly chemical synaptic connections, PSPs that application of $10 \mu$ m curare (D-TC). After curare wash, PSPs returned to their original levels $[n=$ 8; traces represent pre, during (flat) and post application]. Vertical bar equals $10 \mathrm{mV}$, horizontal bar equals $95 \mathrm{msec}$. B, Neurotransmitter release from neuron 110 onto neuron 19 results in an inhibitory PSP. Electrodes filled with 1.5 м KAc were used to perform recordings from 19-110 pairs. Under these recording conditions, hyperpolarizing PSPs were detected in neuron 19 in response to APs evoked in neuron 110 . Vertical bar equals $5 \mathrm{mV}$, horizontal bar equals $95 \mathrm{msec}$. C, 19-110 pairs were paired into contact and cultured for $24 \mathrm{hr}$ in CM containing $10 \mu \mathrm{M} \mathrm{D}-\mathrm{TC}, 10$ $\mu$ m Hex, or $10 \mu$ m vehicle (Co). A significant enhancement of electrical coupling was seen both in the presence of curare $\left({ }^{*} p<0.0005\right)$ and hexamethonium bromide $(t p<0.005)$ compared with controls. (ECCs: $\mathrm{Co}=0.30 \pm 0.06, n=9 ; \mathrm{D}-\mathrm{TC}=0.73 \pm 0.06, n=6$; Hex $=0.59 \pm$ $0.05, n=8)$. Histogram represents mean \pm SEM.

\section{Chronic block of chemical transmission enhances electrical communication}

Based on these previous results, we hypothesized that if electrical and chemical synaptic connections were mutually suppressive at these synapses, the disruption of chemical transmission should facilitate the formation of electrical coupling. To test this, we first determined the nature of the chemical synapse from neuron 110 onto neuron 19. Bath application of the acetylcholine receptor blockers curare (D-TC) and hexamethonium bromide (Hex) eliminated PSPs in neuron 19 evoked by stimulation of presynaptic neuron 110. After a 5 min wash with DM, the amplitude of the PSP returned to pretreatment levels (Fig. 7A). Nearly identical results were achieved in Hex (data not shown), demonstrating the cholinergic nature of this unidirectional chemical connection. Additionally, elimination of PSPs in DM pairs caused by application of cholinergic antagonists confirmed the paucity of electrical coupling at these synapses.

To further examine the nature of these cholinergic synapses, the $1.5 \mathrm{M} \mathrm{KCl}$ solution in the recording pipette was replaced with a 1.5 M KAc solution. Use of a relatively low-resistance $\mathrm{KCl}$ electrode alters the electrical driving force on chloride-based inhibitory currents, thus reversing the polarity of these potentials. PSPs recorded using KAc-filled electrodes after stimulation of neuron 110 were hyperpolarizing or inhibitory (Fig. 7B). Similar inhibitory cholinergic synapses formed by other Helisoma neurons have been reported previously (Haydon, 1988).

Having determined that chemical synapses at 19-110 cell pairs were cholinergic and inhibitory, the effect of chronically blocking nicotinic receptors could be examined. Cells were cultured for 1 or $5 \mathrm{~d}$ in control, curare, or hexamethonium bromide, and ECCs were assessed (Fig. 7C). Compared with control ECC values, a significant enhancement of electrical coupling was seen in cell pairs cultured in the presence of both curare $(p<0.0005)$ occurred in neuron 19 in response to APs evoked in neuron 110 were eliminated during bath 
and hexamethonium bromide $(p<0.005)$. No significant alteration in electrical coupling was detected in 19-19 pairs treated with these cholinergic antagonists $(p>0.05$; data not shown). Thus, in the absence of chemical synaptic communication in $1 \mathrm{~d}$ heterotypic cell pairs, electrical synaptogenesis was potentiated.

\section{Discussion}

A developmental progression of synaptic connectivity from primarily electrical to predominantly chemical communication was demonstrated at identified synapses formed after axotomy in vivo and isolation into cell culture. The strength of electrical synaptic connectivity was inversely related to that of chemical synaptic connections. Over a 5 d period, homotypic Helisoma 19-19 and 110-110 neuronal pairs maintained or strengthened their junctional connectivity during the same time that coupling between heterotypic 19-110 pairs decreased. As this electrical coupling waned, an inhibitory chemical synapse emerged. The time course of this developmental sequence was similar during regeneration in ganglia and in cell culture. It would be interesting to determine whether this sequence of synaptogenesis occurs during normal snail development; however, similar progressions have been demonstrated in other models (Personius et al., 2001; Curtin et al., 2002).

When strong electrical connections form between neurons in culture, chemical connectivity can become masked by junctional current flow and be difficult to quantify (Liu and Nicholls, 1989; Bem et al., 2002). We therefore examined PSP amplitudes at 95 msec after the peak of presynaptic action potentials. Although this approach likely underestimated peak amplitudes of chemical PSPs, it faithfully distinguished chemical from electrical contributions where connections were mixed. In addition, ECC-based predictions $\left(V_{\mathrm{p}}\right)$ match actual values recorded from neuronal pairs at synapses where chemical synaptic connectivity was never detected, demonstrating that $95 \mathrm{msec}$ is a time point at which accurate discrimination of electrical and chemical contributions to synaptic communication can be made.

When the formation of electrical synaptic connectivity was suppressed by the deprivation of ganglia-derived trophic factors, the rate of chemical synapse formation was markedly enhanced. Fluorescent imaging of dye-coupling and vesicle cycling supported electrophysiological data and indicated an inverse correlation between electrical and chemical synaptogenic events. This inverse relationship suggests a mechanism whereby transient intracellular coupling delays the onset of chemical communication at mixed synapses. Many theories have been advanced as to the exact nature of the relationship between electrical and chemical synapse formation early in development. Fischbach (1972) hypothesized the existence of a causal relationship because electrical synapses often form before chemical connections during development. However, in some invertebrates, like the crayfish, a "chemical-like" synapse has been shown to precede and give way to formation of an electrical synapse (Leitch et al., 1989). In vertebrates, expression of specific connexins decreases early in development (Rorig and Sutor, 1996; Chang et al., 1999, 2000; Nadarajah and Parnavelas, 1999) at the onset of chemical synaptogenesis (Kandler and Katz, 1998). In many cases, the formation of the two types of synaptic connectivity is precisely timed, and they do not appear to coexist for long (for review, see Leitch, 1992). Recently, it has been hypothesized that the formation of junctionally coupled "neuronal domains" might help guide the formation, structure, and function of emerging chemically transmitting neural circuits (Yuste et al., 1992).

Haydon and Kater (1988) demonstrated that early junctional connectivity was not required for the formation of chemical synaptic connectivity between specific Helisoma neurons. Indeed, using the highly controlled approach of somatic synapses, the chemical connections studied here formed earlier and more strongly in the absence of electrical coupling. Therefore, one of the implications of transient electrical coupling during development is that specific chemical synapses, perhaps at discrete locations, might be delayed in their formation. At contact sites free of the exclusionary influences of gap junctional coupling, chemical synaptic transmission could develop unfettered.

The question of what happens ultrastructurally to chemical synapses in the presence of junctional coupling remains unclear. Nevertheless, we hypothesize three possible synaptogenic mechanisms to explain the inhibition of chemical synaptic connectivity. First, based on a physical exclusion hypothesis, junctional machinery present in the synaptic membrane could interfere with the incorporation of presynaptic secretory machinery into this same area. That is, the spatial requirements of gap junctional plaques might exclude the placement of chemical synaptic machinery and thus directly hinder the formation of the chemical connections.

A second mechanism might be explained by a functional exclusion hypothesis. Here, molecules necessary for functional synaptic transmission would be depleted because of the presence of electrical coupling. Action potential-evoked increases in intercellular calcium, for example, might be diminished if sufficient levels of presynaptic calcium are lost through gap junctions into the postsynaptic cytoplasm. Such a molecular sink would decrease local concentrations and reduce the probability of action potential-evoked neurotransmitter release. Indeed, morphologically mixed synapses and sites of colocalized chemical and electrical synaptic machinery, are not necessarily indicative of dual transmission (Leitch, 1992; Lin and Faber, 1988).

A third mechanism explaining inhibition of synaptogenesis might involve the exclusion of secretion machinery through the transient disruption of crucial developmental events. In this hypothesis, signaling pathways directly conducted through gap junctions could inhibit the maintenance of the chemical synaptic machinery. Alternatively, intrinsic developmental programs orchestrating the formation of electrical and chemical synapses might involve mutually exclusive molecular cascades. Here, transcriptional or posttranscriptional regulatory pathways might select for the production of one type of synaptic machinery over the other.

These hypotheses are supported, and at the same time complicated, by the finding that pharmacological blockade of acetylcholine receptors results in stronger, longer-lasting electrical connectivity. This indicates a developmental linkage between these two types of synapses that, in some cases such as this, are in direct competition. This competition might be either for space or a functional resource, resulting in the maximization of one type of communication in a manner that is mutually exclusive of maximizing the other. However, the developmental consequences of delaying one form of communication in preference for another remain to be elucidated.

\section{Neurotrophic effects on synaptic development}

The effects of nerve growth and nerve growth-promoting factors on the formation of electrical coupling have been extensively examined. In vertebrates, growth factors have been shown to selectively diminish (Maldonado et al., 1988; Madhukar et al., 1989; Lau et al., 1992) or enhance (Pepper et al., 1989; Nadarajah et al., 1998; Reuss et al., 1998; Aberg et al., 2000) coupling and 
connexin expression. In Helisoma, it is believed that trophic factors are among the substances released from isolated ring ganglia when conditioning defined medium (Wong et al., 1981, 1983, 1984; Barker et al., 1982). In the current studies, a comparison of the developmental sequences in CM versus DM provides the basis for conclusions concerning the role of trophic factors. The formation of electrical coupling at somatic synapses was correlated with the presence of these trophic factors, because neurons paired in DM are coupled more weakly than those in CM. Synapses in DM possessed enhanced maturation of chemical synaptic transmission, and these observations target the developmental mechanism whereby the transient expression of a specific neurotrophin promotes electrical coupling and concomitantly delays chemical synaptogenesis at sites of mixed transmission within the emerging neural network. This developmental mechanism, in the specific case of 19-110 connections, would serve as a functional switch from synchronous to asynchronous activity once electrical coupling is diminished.

Does the presence of trophic factors directly alter chemical communication? Upon initial 19-110 soma-soma contact, when electrical coupling has not yet developed, evoked release from neuron 110 occurs in the continuing presence of trophic factors. Thus, it is not likely that trophic factors alone are the direct cause of the later decrease in chemical synaptic transmission in CMcultured cell pairs. In addition, spontaneous synaptic transmission was not significantly different between CM- and DMcultured somatic synapses. We therefore conclude that delays in chemical synaptic communication are not directly linked to trophic factor influences but are likely mechanistically linked to the emergence of intracellular electrical or metabolic coupling.

Many questions remain concerning the role of transient junctional communication in early synaptic development. For example, what developmental events initiate the formation and elimination of transient intercellular coupling events? What mechanisms underlie the inverse relationship between modes of synaptic transmission? Are these mechanisms cell-intrinsic, or are they products of more complex intercellular signal transduction cascades? Goodman and Spitzer (1979) have questioned whether the function of electrical connectivity can be found, most importantly, in its presence or in its absence. The course of synaptic development presented here adds to their query regarding the role of electrical communication: its transience.

\section{References}

Aberg ND, Carlsson B, Rosengren L, Oscarsson J, Isaksson OG, Ronnback L, Eriksson PS (2000) Growth hormone increases connexin-43 expression in the cerebral cortex and hypothalamus. Endocrinology 141:3879-3886.

Barker DL, Wong RG, Kater SB (1982) Separate factors produced by the CNS of the snail Helisoma stimulate neurite outgrowth and choline metabolism in cultured neurons. J Neurosci Res 8:419-432.

Bem T, Le Feuvre Y, Simmers J, Meyrand P (2002) Electrical coupling can prevent expression of adult-like properties in an embryonic neural circuit. J Neurophysiol 87:538-547.

Bennett MV (1997) Gap junctions as electrical synapses. J Neurocytol 26:349-366.

Bennett MVL (1977) Electrical transmission: a functional analysis and comparison to chemical transmission. In: Handbook of physiology (Kandel ER, ed), pp 357-416. Bethesda: American Physiological Society.

Betz WJ, Mao F, Bewick GS (1992) Activity-dependent fluorescent staining and destaining of living vertebrate motor nerve terminals. J Neurosci 12:363-375.

Bittman K, Owens DF, Kriegstein AR, LoTurco JJ (1997) Cell coupling and uncoupling in the ventricular zone of developing neocortex. J Neurosci 17:7037-7044.

Bodmer R, Verselis V, Levitan IB, Spray DC (1988) Electrotonic synapses between Aplysia neurons in situ and in culture: aspects of regulation and measurements of permeability. J Neurosci 8:1656-1670.

Carrow GM, Levitan IB (1989) Selective formation and modulation of electrical synapses between cultured Aplysia neurons. J Neurosci 9:3657-3664.

Chang Q, Gonzalez M, Pinter MJ, Balice-Gordon RJ (1999) Gap junctional coupling and patterns of connexin expression among neonatal rat lumbar spinal motor neurons. J Neurosci 19:10813-10828.

Chang Q, Pereda A, Pinter MJ, Balice-Gordon RJ (2000) Nerve injury induces gap junctional coupling among axotomized adult motor neurons. J Neurosci 20:674-684.

Connors BW, Benardo LS, Prince DA (1983) Coupling between neurons of the developing rat neocortex. J Neurosci 3:773-782.

Curtin KD, Zhang Z, Wyman RJ (2002) Gap junction proteins expressed during development are required for adult neural function in the Drosophila optic lamina. J Neurosci 22:7088-7096.

De-Miguel FF (2000) Steps in the formation of neurites and synapses studied in cultured leech neurons. Braz J Med Biol Res 33:487-497.

Dermietzel R, Traub O, Hwang TK, Beyer E, Bennett MV, Spray DC, Willecke K (1989) Differential expression of three gap junction proteins in developing and mature brain tissues. Proc Natl Acad Sci USA 86:10148-10152.

Diefenbach TJ, Guthrie PB, Stier H, Billups B, Kater SB (1999) Membrane recycling in the neuronal growth cone revealed by FM1-43 labeling. J Neurosci 19:9436-9444.

Fernandez-de-Miguel F, Drapeau P (1995) Synapse formation and function: insights from identified leech neurons in culture. J Neurobiol 27:367-379.

Fischbach GD (1972) Synapse formation between dissociated nerve and muscle cells in low density cell cultures. Dev Biol 28:407-429.

Goldberg JI, Kater SB (1989) Expression and function of the neurotransmitter serotonin during development of the Helisoma nervous system. Dev Biol 131:483-495.

Goodman CS, Spitzer NC (1979) Embryonic development of identified neurones: differentiation from neuroblast to neurone. Nature 280:208-214

Guthrie SC, Gilula NB (1989) Gap junctional communication and development. Trends Neurosci 12:12-16.

Hadley RD, Kater SB (1983) Competence to form electrical connections is restricted to growing neurites in the snail, Helisoma. J Neurosci 3:924-932.

Hadley RD, Wong RG, Kater SB, Barker DL, Bulloch AG (1982) Formation of novel central and peripheral connections between molluscan central neurons in organ cultured ganglia. J Neurobiol 13:217-230.

Hadley RD, Kater SB, Cohan CS (1983) Electrical synapse formation depends on interaction of mutually growing neurites. Science 221:466-468.

Hadley RD, Bodnar DA, Kater SB (1985) Formation of electrical synapses between isolated, cultured Helisoma neurons requires mutual neurite elongation. J Neurosci 5:3145-3153.

Hamakawa T, Feng ZP, Grigoriv N, Inoue T, Takasaki M, Roth S, Lukowiak K, Hasan SU, Syed NI (1999a) Sevoflurane induced suppression of inhibitory synaptic transmission between soma-soma paired Lymnaea neurons. J Neurophysiol 82:2812-2819.

Hamakawa T, Woodin MA, Bjorgum MC, Painter SD, Takasaki M, Lukowiak K, Nagle GT, Syed NI (1999b) Excitatory synaptogenesis between identified Lymnaea neurons requires extrinsic trophic factors and is mediated by receptor tyrosine kinases. J Neurosci 19:9306-9312.

Haydon PG (1988) The formation of chemical synapses between cellcultured neuronal somata. J Neurosci 8:1032-1038.

Haydon PG, Kater SB (1988) The differential regulation of formation of chemical and electrical connections in Helisoma. J Neurobiol 19:636-655.

Haydon PG, Zoran MJ (1991) Chemical synapses in cell culture. In: Cellular neurobiology: a practical approach (Chad J, Wheal H, eds), pp 57-71. New York: Oxford UP.

Janse C, van der Roest M, Slob W (1986) Age-related decrease in electrical coupling of two identified neurons in the mollusc Lymnaea stagnalis. Brain Res 376:208-212.

Kandler K, Katz LC (1998) Relationship between dye coupling and spontaneous activity in developing ferret visual cortex. Dev Neurosci 20:59-64.

Kater SB (1974) Feeding in Helisoma trivolvis: the morphological and physiological bases of a fixed action pattern. Am Zool 14:1017-1036.

Kater SB (1985) Determinants of specificity of electrical synapses: the making and breaking of connections in Helisoma. In: Gap junctions (Bennett 
MVL, Spray DC, eds), pp 241-250. Cold Spring Harbor: Cold Spring Harbor Laboratory.

Kay AR, Alfonso A, Alford S, Cline HT, Holgado AM, Sakmann B, Snitsarev VA, Stricker TP, Takahashi M, Wu LG (1999) Imaging synaptic activity in intact brain and slices with FM1-43 in C. elegans, lamprey, and rat. Neuron 24:809-817.

Kristan WB, Eisenhart FJ, Johnson LA, French KA (2001) Development of neuronal circuits and behaviors in the medicinal leech. Brain Res Bull 53:561-570.

Lau AF, Kanemitsu MY, Kurata WE, Danesh S, Boynton AL (1992) Epidermal growth factor disrupts gap-junctional communication and induces phosphorylation of connexin43 on serine. Mol Biol Cell 3:865-874.

Leitch B (1992) Ultrastructure of electrical synapses: review. Electron Microsc Rev 5:311-339.

Leitch B, Cobb JL, Heitler WJ, Pitman RM (1989) Post-embryonic development of rectifying electrical synapses in the crayfish: ultrastructure. J Neurocytol 18:749-761.

Lin J-W, Faber F (1988) Synaptic transmission mediated by single club endings on the goldfish Mauthner cell. I. Characteristics of electrotonic and chemical postsynaptic potentials. J Neurosci 8:1302-1312.

Liu Y, Nicholls J (1989) Steps in the development of chemical and electrical synapses by pairs of identified leech neurons in culture. Proc R Soc Lond B Biol Sci 236:253-268.

Lo Turco JJ, Kriegstein AR (1991) Clusters of coupled neuroblasts in embryonic neocortex. Science 252:563-566.

Madhukar BV, Oh SY, Chang CC, Wade M, Trosko JE (1989) Altered regulation of intercellular communication by epidermal growth factor, transforming growth factor-beta and peptide hormones in normal human keratinocytes. Carcinogenesis 10:13-20.

Magoski NS, Bulloch AG (1998) Trophic and contact conditions modulate synapse formation between identified neurons. J Neurophysiol 79:3279-3283.

Maldonado PE, Rose B, Loewenstein WR (1988) Growth factors modulate junctional cell-to-cell communication. J Membr Biol 106:203-210.

Mentis GZ, Diaz E, Moran LB, Navarrete R (2002) Increased incidence of gap junctional coupling between spinal motoneurones following transient blockade of NMDA receptors in neonatal rats. J Physiol (Lond) 544:757-764.

Murphy AD (2001) The neuronal basis of feeding in the snail, Helisoma, with comparisons to selected gastropods. Prog Neurobiol 63:383-408.

Nadarajah B, Parnavelas JG (1999) Gap junction-mediated communication in the developing and adult cerebral cortex. Novartis Found Symp 219:157-170.

Nadarajah B, Thomaidou D, Evans WH, Parnavelas JG (1996) Gap junctions in the adult cerebral cortex: regional differences in their distribution and cellular expression of connexins. J Comp Neurol 376:326-342.

Nadarajah B, Makarenkova H, Becker DL, Evans WH, Parnavelas JG (1998) Basic FGF increases communication between cells of the developing neocortex. J Neurosci 18:7881-7890.

Nicholls JG, Liu Y, Payton BW, Kuffler DP (1990) The specificity of synapse formation by identified leech neurones in culture. J Exp Biol 153:141-154.

Owens DF, Kriegstein AR (1998) Patterns of intracellular calcium fluctuation in precursor cells of the neocortical ventricular zone. J Neurosci 18:5374-5388.
Pastor AM, Mentis GZ, De La Cruz RR, Diaz E, Navarrete R (2003) Increased electrotonic coupling in spinal motoneurons after transient botulinum neurotoxin paralysis in the neonatal rat. J Neurophysiol 89:793-805.

Peinado A, Yuste R, Katz LC (1993a) Extensive dye coupling between rat neocortical neurons during the period of circuit formation. Neuron 10:103-114.

Peinado A, Yuste R, Katz LC (1993b) Gap junctional communication and the development of local circuits in neocortex. Cereb Cortex 3:488-498.

Pepper MS, Spray DC, Chanson M, Montesano R, Orci L, Meda P (1989) Junctional communication is induced in migrating capillary endothelial cells. J Cell Biol 109:3027-3038.

Personius KE, Balice-Gordon RJ (2001) Loss of correlated motor neuron activity during synaptic competition at developing neuromuscular synapses. Neuron 31:395-408.

Personius K, Chang Q, Bittman K, Panzer J, Balice-Gordon R (2001) Gap junctional communication among motor and other neurons shapes patterns of neural activity and synaptic connectivity during development. Cell Commun Adhes 8:329-333.

Poyer JC, Zoran MJ (1996) Activity-dependent induction of functional secretory properties at cultured neuromuscular synapses of Helisoma. J Neurophysiol 76:2635-2643.

Ready DF, Nicholls J (1979) Identified neurones isolated from leech CNS make selective connections in culture. Nature 281:67-69.

Reuss B, Dermietzel R, Unsicker K (1998) Fibroblast growth factor 2 (FGF-2) differentially regulates connexin (cx) 43 expression and function in astroglial cells from distinct brain regions. Glia 22:19-30.

Rorig B, Sutor B (1996) Regulation of gap junction coupling in the developing neocortex. Mol Neurobiol 12:225-249.

Rozental R, Morales M, Mehler MF, Urban M, Kremer M, Dermietzel R, Kessler JA, Spray DC (1998) Changes in the properties of gap junctions during neuronal differentiation of hippocampal progenitor cells. J Neurosci 18:1753-1762.

Wong RG, Hadley RD, Kater SB, Hauser GC (1981) Neurite outgrowth in molluscan organ and cell cultures: the role of conditioning factor(s). J Neurosci 1:1008-1021.

Wong RG, Martel EC, Kater SB (1983) Conditioning factor(s) produced by several molluscan species promote neurite outgrowth in cell culture. J Exp Biol 105:389-393.

Wong RG, Barker DL, Kater SB, Bodnar DA (1984) Nerve growthpromoting factor produced in culture media conditioned by specific CNS tissues of the snail Helisoma. Brain Res 292:81-91.

Woodin MA, Hamakawa T, Takasaki M, Lukowiak K, Syed NI (1999) Trophic factor-induced plasticity of synaptic connections between identified Lymnaea neurons. Learn Mem 6:307-316.

Yuste R, Peinado A, Katz LC (1992) Neuronal domains in developing neocortex. Science 257:665-669.

Zoran MJ, Haydon PG, Matthews PJ (1989) Aminergic and peptidergic modulation of motor function at an identified neuromuscular junction in Helisoma. J Exp Biol 142:225-243.

Zoran MJ, Metts BA, Poyer JC (1996) Specific muscle contacts induce increased transmitter release and neuritic arborization in motoneuronal cultures. Dev Biol 179:212-222. 